\title{
A certificateless public key encryption based return routability protocol for next-generation IP mobility to enhance signalling security and reduce latency
}

\author{
SENTHILKUMAR MATHI*I, R NIVETHA, B PRIYADHARSHINI and S PADMA \\ Department of Computer Science and Engineering, Amrita University, Coimbatore, India \\ e-mail: m_senthil@cb.amrita.edu; ravichandran_nivetha@rediffmail.com; bpriya091@gmail.com; \\ spadma93@yahoo.com
}

MS received 21 August 2015; revised 9 December 2016; accepted 5 May 2017; published online 16 November 2017

\begin{abstract}
IPv6 mobility is an IETF standard that has added roaming capabilities of mobile node (MN). It allows MNs to travel from one network to another without any distraction in communication service. MNs register their current location to home stations and correspondent hosts via a process known as binding update. In IPv6 mobility, return routability protocol (RRP) is a standard procedure for updating the current location of MNs through binding update message to their communicants. However, RRP has several security threats and issues. Subsequently, RRP was integrated with identity-based encryption for improvement of security. Nevertheless, it suffers from some limitations such as inherent key escrow problem, lack of key revocation, high computational load and latency while providing security. Hence, this paper proposes a novel approach called optimised RRP using certificateless public key encryption to address these issues. The proposed protocol is simulated and validated using Automated Validation of Internet Security Protocols and Applications (AVISPA) - a model checker. Finally, the simulation and numerical results illustrate the extent to which the proposed protocol surpasses the existing method in terms of enhanced security and significant reduction in communication payload with minimised latency.
\end{abstract}

Keywords. Binding update; confidentiality; authentication; return routability and mutual authentication.

\section{Prologue}

Internet protocol (IP) is an internet layer protocol that helps in delivering packets from a source to a destination host based on the IP address accessible in the packet header. Mobile IP is a communication protocol that allows mobile users associated with one network to stay connected even while moving to a different network with a different IP address [1, 2]. IPv6 mobility, also known as Mobile IPv6 (MIPv6), allows the mobile node (MN) to remain connected to a correspondent node $(\mathrm{CN})$ even when the $\mathrm{MN}$ moves from a home network to another network. The principal elements of MIPv6 include mobile node (MN), home agent (HA) and correspondent node $(\mathrm{CN})$. In the environment of IPv6 mobility, the MN obtains a Home-of address (HoA) from its original location with an initial registration. Additionally, it acquires a temporary address known as Care-of address (CoA) when it moves to a foreign network [3]. The CoA is registered to HA by MN. When a datagram is sent from $\mathrm{CN}$ to $\mathrm{MN}$, it will be passed to the MN's HA. In turn, HA checks if the MN is in the home or

*For correspondence foreign network [4]. It then forwards the datagram to HoA of MN when it is in the home network and to CoA when it is in the foreign network.

Over the years, a number of BU schemes [5-8] were proposed. The base scheme known as Return Routability Protocol (RRP) is a binding update procedure that allows a node to confirm the presence of another node to which the packet is sent [9]. In order to overcome the various security threats faced by RRP, Return Routability using IdentityBased Encryption (RR-IBE) was suggested [10]. In RRIBE that uses public key cryptography, private key is obtained from a trusted third party called Private Key Generator (PKG). However, this approach introduces the inherent key escrow problem and lack of key revocation $[11,12]$. To overcome the disadvantages of RR-IBE, the current paper proposes an improved RRP that uses Certificate Less-Public Key Encryption (CL-PKE) [13].

The rest of the paper is organised as follows: Section 2 briefly explains the related works. Section 3 presents a brief review of the preliminaries required for the proposed protocol. Section 4 presents the security analysis of the proposed protocol. Section 5 verifies the security properties of the proposed protocol by formal analysis using AVISPA. 
Section 6 discusses the performance of our protocol with existing protocols in terms of communication payload and latency of BU.

\section{Related works}

\subsection{Return routability protocol}

The RRP mechanism sends BU messages between the mobile and correspondent host as shown in figure 1. It consists of the following messages: Home-of Initiation (HoTI), Care-of Test Initiation (CoTI), Home-of Test (HoT), and Care-of Test (CoT). Here, the MN sends HoTI and CoTI to CN in two modes: directly and through home agent. The communication link MN-HA is secured through IP security (IPSec) tunnelling. Then, CN receives the HoTI from the HA and the CoTI message from the MN. In turn, the $\mathrm{CN}$ sends HoT and CoT messages to MN by two methods: it sends the message directly or via base station. The RRP culminates the procedure when MN receives both the messages. However, the traditional RRP suffers from various security attacks such as replay, man-in-the-middle, reflection and amplification attacks. Hence, it is necessary to enhance the RRP to surmount these issues.

\subsection{Return routability protocol with identity-based encryption}

Identity-based encryption (IBE) is incorporated with RRP. This approach employs a trusted third party called the Private Key Generator (PKG). The PKG maintains a private key for a correspondent host. The RR-IBE provides authentication, confidentiality, integrity and attack prevention. Though RR-IBE has advantages, the use of PKG to generate the private key introduces an inherent key escrow problem. The PKG can falsify an entity's signature in an

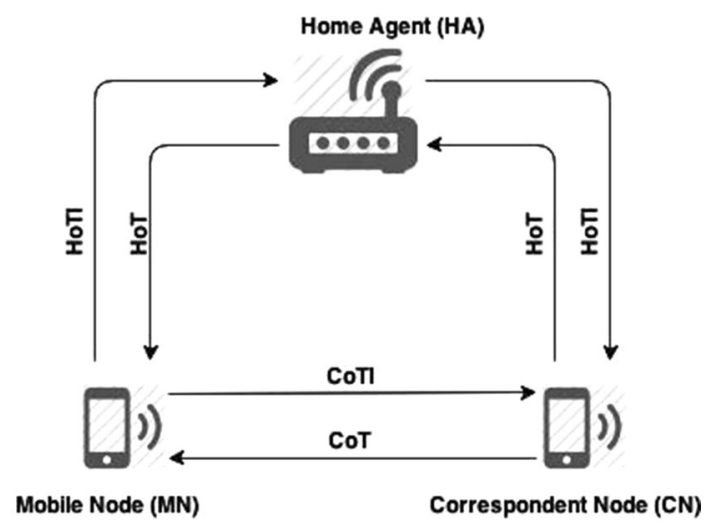

Figure 1. Return routability protocol. identity-based signature method, thus the RR-IBE protocol cannot offer true non-repudiation. In addition, IBE in its most basic form lacks in key revocation.

\section{Proposed protocol}

The proposed protocol with CLPKE uses a trusted third party known as Key Generation Centre (KGC). In contrast to the PKG in RR-IBE, the KGC does not have access to private key of the entities. Instead, the KGC supplies the entity with a partial private key. The proposed protocol is based on the following assumptions:

- The MN first registers with HA and $\mathrm{CN}$ to acquire the public keys.

- There exists a secure IPSec tunnel between MN and HA.

- The path between $\mathrm{CN}$ and KGC is secured because KGC authenticates each of the registered communicants.

\subsection{Phases of key generation}

Key generation of the proposed approach consists of five phases. Here, let a security parameter $k$ be given as an input to the setup phase [12].

1. Preliminary setup: The setup phase is executed by the $\mathrm{KGC}$ and the output is distributed to the $\mathrm{MN}, \mathrm{CN}$ and HA. It operates as follows,

- Run a Bilinear Diffie-Hellman parameter generator algorithm with input $k$ and generate the output as $\left(\mathrm{G}_{1}, \mathrm{G}_{2}, e\right)$, where $e$ is a bilinear map such that $e: \mathrm{G}_{1}$ $\times \mathrm{G}_{1} \rightarrow \mathrm{G}_{2}$. Here, $\mathrm{G}_{1}$ and $\mathrm{G}_{2}$ denote an additive group and a multiplicative group of some prime order $q$ respectively.

- Select an arbitrary generator P of group $\mathrm{G}_{1}$.

- Choose a master key $s$ at random from $\mathrm{Z}_{q}^{*}$ and $\mathrm{P}_{0}=$ s.P

The output generated from the setup phase is $\left(\mathrm{G}_{1}\right.$, $\mathrm{G}_{2}$, e, $\mathrm{n}, \mathrm{P}, \mathrm{P}_{0}, \mathrm{H}_{1}, \mathrm{H}_{2}, \mathrm{H}_{3}, \mathrm{H}_{4}$ ), called as params, where $n$ is the length of the plaintext; $\mathrm{H}_{1}, \mathrm{H}_{2}, \mathrm{H}_{3}$, and $\mathrm{H}_{4}$ are hash functions and defined as follows,

$$
\begin{aligned}
& \mathrm{H}_{1}:\{0,1\}^{*} \rightarrow \mathrm{G}_{1}^{*} \\
& \mathrm{H}_{2}: \mathrm{G}_{2} \rightarrow\{0,1\}^{\mathrm{n}} \\
& \mathrm{H}_{3}:\{0,1\}^{\mathrm{n}} \times\{0,1\}^{\mathrm{n}} \rightarrow \mathrm{Z}_{\mathrm{q}}^{*} \\
& \mathrm{H}_{4}:\{0,1\}^{\mathrm{n}} \rightarrow\{0,1\}^{\mathrm{n}} .
\end{aligned}
$$

2. Extraction of partial private key: The KGC runs this phase to produce a partial private key of $\mathrm{CN}$. It uses an identifier of $\mathrm{CN}\left(\mathrm{ID}_{\mathrm{CN}}\right)$ as input and generates the partial private key as follows, 


$$
\mathrm{D}_{\mathrm{CN}}=\mathrm{s} . \mathrm{H}_{1}\left(\mathrm{ID}_{\mathrm{CN}}\right)
$$

3. Generation of secret value: This phase is executed by $\mathrm{MN}, \mathrm{CN}$ and HA separately that takes params and their respective IDs (i.e., $\mathrm{ID}_{\mathrm{MN}}, \mathrm{ID}_{\mathrm{CN}}$ and $\mathrm{ID}_{\mathrm{HA}}$ ) as inputs. The entities select secret values at random and gives output $\mathrm{X}_{\mathrm{MN}}, \mathrm{X}_{\mathrm{CN}}, \mathrm{X}_{\mathrm{HA}}$ for $\mathrm{MN}, \mathrm{CN}$ and $\mathrm{HA}$, respectively.

4. Generation of private key: The $\mathrm{CN}$ generates the private key $\mathrm{R}_{\mathrm{CN}}$ using the params, secret value $\mathrm{X}_{\mathrm{CN}}$ and partial private key $\mathrm{D}_{\mathrm{CN}}$ obtained from KGC. It is computed as follows:

$$
\mathrm{R}_{\mathrm{CN}}=\mathrm{X}_{\mathrm{CN}} \cdot \mathrm{D}_{\mathrm{CN}}
$$

5. Generation of public key: This phase is run by $\mathrm{CN}$. The inputs are params and its respective secret value $\mathrm{X}_{\mathrm{CN}}$. It computes the public key of $\mathrm{CN}, \mathrm{P}_{\mathrm{CN}}=\left(\mathrm{A}_{\mathrm{CN}}, \mathrm{B}_{\mathrm{CN}}\right)$, where $\mathrm{A}_{\mathrm{CN}}=\mathrm{X}_{\mathrm{CN}} \mathrm{P}$ and $\mathrm{B}_{\mathrm{CN}}=\mathrm{X}_{\mathrm{CN}} \mathrm{P}_{0}$.

\subsection{Encryption and decryption}

This section discusses the encryption and decryption.

Encryption: To encrypt a message $\mathrm{M} \in\{0,1\}^{\mathrm{n}}$ by any entity, say $\mathrm{CN}$ with $\mathrm{ID}_{\mathrm{CN}}$ and its public key $\mathrm{P}_{\mathrm{CN}}$, the following steps are performed:

- Check if $A_{C N}, B_{C N} \in G_{1}^{*}$ and $e\left(A_{C N}, P_{0}\right)=e\left(B_{C N}, P\right)$.

- Let $\mathrm{Q}_{\mathrm{CN}}=\mathrm{H}_{1}\left(\mathrm{ID}_{\mathrm{CN}}\right) \in \mathrm{G} 1^{*}$

- Select a value $\sigma$ at random such that $\sigma \in\{0,1\}^{\mathrm{n}}$.

- Let $\mathrm{r}=\mathrm{H}_{3}(\sigma, \mathrm{M})$.

- Compute and output the ciphertext $\mathrm{C}=(\mathrm{rP}, \operatorname{XOR}(\sigma$, $\left.\mathrm{H}_{2}\left(\mathrm{e}\left(\mathrm{Q}_{\mathrm{CN}}, \mathrm{B}_{\mathrm{CN}}\right) \mathrm{r}\right)\right)$, XOR(M, $\left.\left.\mathrm{H}_{4}(\sigma)\right)\right)$ such that $\mathrm{C}=\mathrm{G}_{1}$ $\times\{0,1\}^{\mathrm{n}}$

Decryption: Decryption of $\mathrm{C}$ can be done by $\mathrm{CN}$ using its private key $\mathrm{R}_{\mathrm{CN}}$ as follows,

- Compute XOR $\left(\mathrm{XOR}\left(\sigma, \mathrm{H}_{2}\left(\mathrm{e}\left(\mathrm{Q}_{\mathrm{CN}}, \mathrm{B}_{\mathrm{CN}}\right) \mathrm{r}\right)\right), \mathrm{H}_{2-}\right.$ $\left.\left(\mathrm{e}\left(\mathrm{R}_{\mathrm{CN}}, \mathrm{rP}\right)\right)\right)=\sigma$.

- Compute XOR $\left.\left(\mathrm{XOR}\left(\mathrm{M}, \mathrm{H}_{4}(\sigma)\right)\right), \mathrm{H}_{4}\left(\sigma^{\prime}\right)\right)=\mathrm{M}^{\prime}$.

- Set $\mathrm{r}^{\prime}=\mathrm{H}_{3}\left(\sigma^{\prime}, \mathrm{M}^{\prime}\right)$.

- Output $\mathrm{M}^{\prime}$ as the decryption of $\mathrm{C}$. When $\mathrm{C}$ is a valid ciphertext of $\mathrm{M}$ using $\mathrm{P}_{\mathrm{CN}}$ and $\mathrm{ID}_{\mathrm{CN}}$, then deciphering of $\mathrm{C}$ will result in an output $\mathrm{M}^{\prime}=\mathrm{M}$.

\subsection{Proposed protocol description}

The pictorial representation of the proposed protocol is shown in figure 2.

Table 1 shows the notations used in the proposed protocol. The intricacies of the proposed message flows are as follows:

Initially, the MN sends the HOTI message to $\mathrm{CN}$ through the HA using IPSec tunnel mode. The message M1 contains the source addresses $\left(\mathrm{MN}_{\mathrm{COA}}\right.$ and $\left.\mathrm{MN}_{\mathrm{HOA}}\right)$ of the $\mathrm{MN}$ and the destination address of the HA $\left(\mathrm{MN}_{\mathrm{HAA}}\right)$.

Here, M1 includes message with the old address of the $\mathrm{MN}\left(\mathrm{MN}_{\mathrm{OldCOA}}\right)$ to inform the HA or $\mathrm{CN}$ of the MN's preceding location before it moves to another network with $\mathrm{MN}_{\mathrm{CoA}}$. Consequently, if a packet is sent from the $\mathrm{CN}$ to the existing $\mathrm{MN}_{\mathrm{COA}}$, the $\mathrm{CN}$ can know how to send this message to the present $\mathrm{MN}_{\mathrm{COA}}$. Both $\mathrm{M} 1$ and M1' contain a nonce or random number $\left(\mathrm{N}_{0}\right)$. On receiving both M1 and M1', the CN compares both the messages. If $\mathrm{N}_{0}$ is same in both, then $\mathrm{CN}$ sends a partial private key request to the KGC. In the HOTI, HOTI', COTI and BU messages of $\mathrm{MN}$, the random numbers are encrypted using certificateless public key encryption. This encryption is done using the public key of $\mathrm{CN}\left(\mathrm{P}_{\mathrm{CN}}\right)$ as mentioned in the previous section. The message flows of the proposed protocol are shown in figure 3 and the details are as follows,

Home-of Test Init:

$$
\mathrm{MN} \rightarrow \mathrm{HA}: \mathrm{M} 1
$$

where $\mathrm{M} 1=\mathrm{MN}_{\mathrm{HOA}}, \mathrm{CN}_{\mathrm{Addr}}, \mathrm{MN}_{\mathrm{OldCOA}},\left\{\mathrm{N}_{0}, \mathrm{MN}_{\mathrm{HOA}}\right.$, $\left.\mathrm{MN}_{\mathrm{COA}}, \mathrm{P}, \mathrm{P}_{0}\right\}$ CLPKE_P $\mathrm{CN}_{\mathrm{N}}$

$$
\left(\mathrm{HOTI}^{\prime}\right) \mathrm{HA} \rightarrow \mathrm{CN}: \mathrm{M}{ }^{\prime}
$$

where $\mathrm{M1}^{\prime}=\mathrm{MN}_{\mathrm{HOA}}, \mathrm{CN}_{\mathrm{Addr}}, \mathrm{MN}_{\mathrm{OldCOA}},\left\{\mathrm{N}_{0}, \mathrm{MN}_{\mathrm{HOA}}\right.$, $\left.\mathrm{MN}_{\mathrm{COA}}, \mathrm{P}, \mathrm{P}_{0}\right\}$ CLPKE_P $\mathrm{P}_{\mathrm{CN}}$

Care-of Test Init:

$$
(\mathrm{COTI}) \mathrm{MN} \rightarrow \mathrm{CN}: \mathrm{M} 2
$$

where $\mathrm{M} 2=\mathrm{ID}_{\mathrm{MN}}, \mathrm{MN}_{\text {OldCOA }},\left\{\mathrm{N}_{0}, \mathrm{MN}_{\mathrm{COA}}, \mathrm{P}\right.$, $\left.\mathrm{P}_{0}\right\}$ CLPKE_P $\mathrm{P}_{\mathrm{CN}}$

Partial private key request:

$$
\text { (PPKReq) } \mathrm{CN} \rightarrow \mathrm{KGC}: \mathrm{M} 3
$$

where $\mathrm{M} 3=\mathrm{CN}_{\mathrm{Addr}}, \mathrm{H}_{1}\left(\mathrm{ID}_{\mathrm{CN}}\right)$, Dreq $\mathrm{CN}_{\mathrm{CN}}$

Authentication of $\mathrm{CN}$ and partial private key response:

$$
\text { (PPKRes) } \mathrm{KGC} \rightarrow \mathrm{CN}: \mathrm{M} 4
$$

where $\mathrm{M} 4=\operatorname{Dres}_{\mathrm{CN}}, \mathrm{H}_{2}\left(\mathrm{CN}_{\mathrm{Addr}}\right), \mathrm{D}_{\mathrm{CN}}$ Authentication of $M N$ :

$$
(\mathrm{AU}) \mathrm{CN} \rightarrow \mathrm{MN}: \mathrm{M} 5
$$

where $\mathrm{M} 5=\mathrm{CN}_{\mathrm{Addr}}, \mathrm{CN}_{\mathrm{COA}}, \mathrm{XOR}\left(\mathrm{N}_{0}, \mathrm{~N}_{1}\right)$

Binding update:

$$
(\mathrm{BU}) \mathrm{MN} \rightarrow \mathrm{CN}: \mathrm{M} 6
$$

where $\mathrm{M} 6=\mathrm{BU}, \mathrm{CN}_{\mathrm{Addr}}, \mathrm{ID}_{\mathrm{MN}},\left\{\mathrm{N}_{1}, \mathrm{MN}_{\mathrm{COA}}, \mathrm{H}_{3}\left[\mathrm{~N}_{1}\right]\right\}-$ CLPKE_ $\mathrm{P}_{\mathrm{CN}}$

Binding Acknowledgment:

$$
(\mathrm{BA}) \mathrm{CN} \rightarrow \mathrm{MN}: \mathrm{M} 7
$$

where $\mathrm{M} 7=\mathrm{BA}, \mathrm{CN}_{\mathrm{CNA}}, \mathrm{CN}_{\mathrm{COA}}, \mathrm{XOR}\left(\mathrm{N}_{0}, \mathrm{~N}_{1}\right)$ 


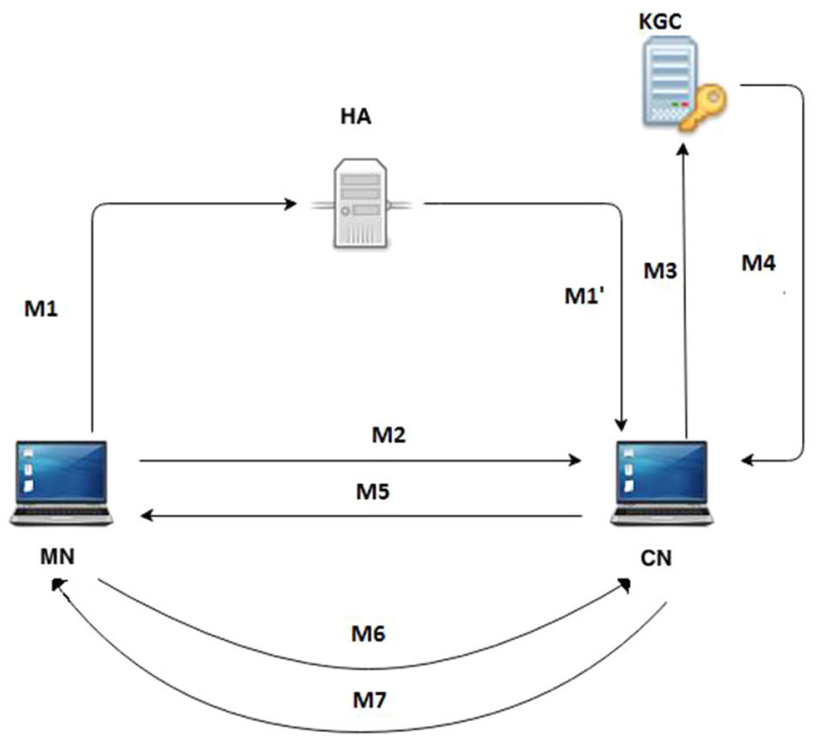

Figure 2. Proposed protocol architecture.

Table 1. Notations used in proposed protocol.

\begin{tabular}{lc}
\hline Symbol & Description \\
\hline $\mathrm{A} \rightarrow \mathrm{B}: \mathrm{M}$ & A sends the message M to B \\
$\{$ Set of fields $\}$ & Encryption of set of fields \\
CLPKE_P & CLKE with the key $\mathrm{P}_{\mathrm{e}}$ \\
Dreq $_{\mathrm{CN}}$ & Partial private key request \\
Dres $_{\mathrm{CN}}$ & Partial private key response \\
$\mathrm{MN}_{\mathrm{COA}}$ and & MN's care-of address and home of address \\
$\mathrm{MN}_{\mathrm{HOA}}$ & MN's old care-of address \\
$\mathrm{MN}_{\mathrm{OldCOA}}$ & MN's home agent address \\
$\mathrm{MN}_{\mathrm{HAA}}$ & Address of the correspondent node \\
$\mathrm{CN}_{\mathrm{Addr}}$ & Nonces \\
$\mathrm{N}_{0}, \mathrm{~N}_{1}$ & Bit patterns indicating binding update and \\
$\mathrm{BU}, \mathrm{BA}$ & acknowledgement \\
$\mathrm{P}_{\mathrm{KCN}}$ & Public key of CN \\
\hline
\end{tabular}

\section{Security analysis}

In this section, security attributes such as authentication, confidentiality, data integrity, inherent key escrow and attack prevention are discussed.

\subsection{Data authentication}

In the proposed protocol, authentication is provided between all the correspondent pairs. Here, an encrypted nonce $\mathrm{N}_{0}$ using CLPKE is sent in the messages M1, M1' and M2. This nonce sent from $\mathrm{MN}$ to $\mathrm{CN}$ can be used for authentication from messages M5 to M7. The CN then authenticates $\mathrm{MN}$ by generating $\mathrm{N}_{1}$ and subsequently performs XOR with $\mathrm{N}_{0}$ and sends it to MN. Further, MN authenticates $\mathrm{CN}$ by sending $\mathrm{N}_{1}$ to $\mathrm{CN}$. In the proposed protocol, KGC also provides authentication for its communicants using hash functions.

\subsection{Data confidentiality and integrity}

The public key $\mathrm{P}_{\mathrm{CN}}$ is used for enciphering in $\mathrm{M} 1, \mathrm{M} 2$ and M6. The secret value $X_{C N}$ used in $P_{C N}$ is generated using the identity of $\mathrm{CN}\left(\mathrm{ID}_{\mathrm{CN}}\right)$ and is known only by $\mathrm{CN}$. M5 depends on the nonce $\mathrm{N}_{0}$ from the prior M1' and M2, where $\mathrm{N}_{0}$ is identified by the mobile and correspondent node. M5 performs XOR with $\mathrm{N}_{0}$ and $\mathrm{N}_{1}$ and, hence, the preliminary message for the scheme uses secure communication through tunnelling. All enciphered packets require a private key for deciphering, making it hard for an impostor to take out any field from the packets. In RR-IBE when the PKG is compromised and the private key is known, the intruder can decrypt and view the messages, whereas in the proposed protocol when the partial private key is compromised, the intruder cannot decrypt the messages. Here, the MN sends two messages (M1 and M2) to CN. The intruder can seize the messages, but cannot decipher or modify the data since these messages are enciphered with the public key of $\mathrm{CN}$ $\left(\mathrm{P}_{\mathrm{CN}}\right)$. The $\mathrm{CN}$ can explore for any modification by comparing M1' with M2. In addition, the impostor cannot generate a fake message since he only recognises the address of $\mathrm{CN}$. Consequently, any alteration to the exchanged packet cannot be possible unless an opponent knows the secret value of $\mathrm{CN}$.

\subsection{Preventing inherent key escrow}

In the RR-IBE protocol, the use of PKG to generate the private key introduces the inherent key escrow problem because if the PKG is compromised with an adversary, then he can decrypt the ciphertext using the private key. To address this issue, the proposed protocol involves KGC to generate only the partial private key. The KGC then distributes the partial private key to $\mathrm{CN}$ (it can generate the private key as discussed in section 3.1). Thus, the proposed protocol prevents the key escrow problem and the attacker from acquiring the private key of $\mathrm{CN}$.

\subsection{Man-in-the-middle attack prevention}

An opponent might lay down himself as the man-in-themiddle (MITM) between a legitimate $\mathrm{MN}$ and the $\mathrm{CN}$ by sending false BUs. However, the proposed protocol prevents MITM attack without revealing any information to the opponent. If he alters the address of the message, the correspondent user can detect it easily since the message contains the original address of MN. In addition, the $\mathrm{CN}$ compares messages M1' and M2. If it finds that their contents are not equivalent, it rejects the message M2. Subsequently, it does not send a message requesting a partial 


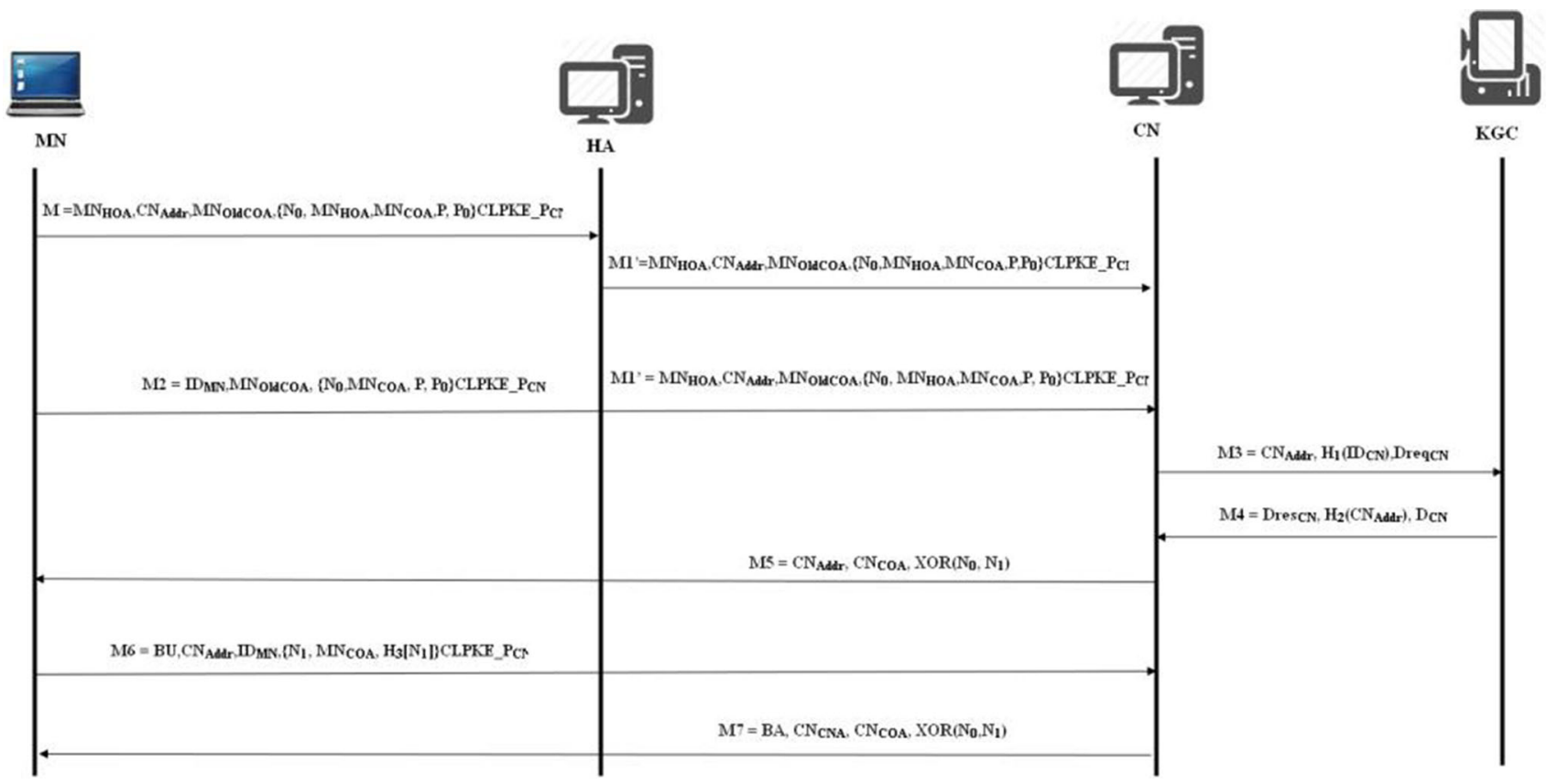

Figure 3. Message sequence diagram of the proposed protocol.

private key from KGC. If an intruder receives M5 from $\mathrm{CN}$, it alters its address, and sends the false address to the mobile user. However, the MN receives M5 and compares it with the message M2 to notice any changes that occurred in M5. As a result, the MN can identify the opponent and prevent the message M6.

\subsection{Replay attack prevention}

The attacker can monitor the communication channel, record the current run of the message, and replay it later. Suppose the intruder captures M1 from HA and sends it to $\mathrm{CN}$ without altering its fields. Here in the proposed protocol, $\mathrm{CN}$ compares $\mathrm{M} 2$ with $\mathrm{M} 1$ '. If $\mathrm{M} 1$ ' and $\mathrm{M} 2$ are found equivalent, it obtains the partial private key from KGC to generate the private key $\mathrm{R}_{\mathrm{CN}}$ to decrypt the message.

If the attacker sends both M1' and M2 to the $\mathrm{CN}$, it receives the partial private key from the KGC and acquires $\mathrm{N}_{0}$, which is recognised through the legitimate $\mathrm{MN}$ and $\mathrm{CN}$. The $\mathrm{CN}$ sends a message M5 to the intruder that contains the XOR value of $\mathrm{N}_{0}$ with a fresh random number $\mathrm{N}_{1}$. Here, the possible replay is prevented since the attacker does not know $\mathrm{N}_{0}$.

\subsection{Amplification attack prevention}

Generating more than one message from an original message and sending to all the nodes is called an amplification attack. Here, the attacker sends a message from $\mathrm{CN}$ to $\mathrm{MN}$ via HA to send more number of messages to other nodes. It is prevented in the proposed protocol since no messages or data are sent from $\mathrm{CN}$ to the home network in our protocol. In addition, KGC verifies the authentication of all the communicants. Table 2 lists the security analysis of the proposed protocol with existing protocols.

\section{Formal analysis using AVISPA}

In this section, a formal analysis of the proposed protocol is presented using Automated Validation of Internet Security Protocols and Applications (AVISPA) to validate the security properties $[14,15]$. AVISPA is basically a security tool that provides High-Level Protocol Specification Language (HLPSL) for describing the properties of security protocols. The written HLPSL is then translated into Intermediate Format (IF). Subsequently, the translated IF is fed into the following back-ends of AVISPA for automatic analysis to detect the attacks.

1. On-the-fly Model-Checker (OFMC)

2. Constraint - Logic-based Attack Searcher (CL-AtSe)

3. SAT-based Model-Checker (SATMC)

4. Tree Automata based on Automatic Approximations for the Analysis of Security Protocols (TA4SP).

In AVISPA, the entities of the model are defined by a role. The proposed protocol is specified with the role definition of the entities for MN, HA and CN. The role definition of 
Table 2. Security analysis.

\begin{tabular}{|c|c|c|c|c|c|c|}
\hline & \multicolumn{3}{|c|}{ MN-HA } & \multicolumn{3}{|c|}{$\mathrm{MN}-\mathrm{CN}$} \\
\hline & $\mathrm{RR}$ & $\begin{array}{l}\text { RR- } \\
\text { IBE }\end{array}$ & Proposed & RR & $\begin{array}{l}\text { RR- } \\
\text { IBE }\end{array}$ & Proposed \\
\hline Authentication & Yes & No & Yes & No & Yes & Yes \\
\hline $\begin{array}{l}\text { Data } \\
\text { confidentiality }\end{array}$ & Yes & Yes & Yes & No & Yes & Yes \\
\hline Data integrity & Yes & Yes & Yes & No & No & Yes \\
\hline $\begin{array}{l}\text { Preventing } \\
\text { inherent key } \\
\text { escrow }\end{array}$ & No & No & Yes & No & No & Yes \\
\hline $\begin{array}{l}\text { MITM attack } \\
\text { prevention }\end{array}$ & No & Yes & Yes & No & No & Yes \\
\hline $\begin{array}{l}\text { Replay attack } \\
\text { prevention }\end{array}$ & No & Yes & Yes & No & Yes & Yes \\
\hline $\begin{array}{l}\text { Amplification } \\
\text { attack } \\
\text { prevention }\end{array}$ & No & Yes & Yes & No & Yes & Yes \\
\hline
\end{tabular}

an entity contains the variables and set of transitions. The sample role definition of $\mathrm{MN}$ for the proposed protocol is shown in figure 4. Once the HLPSL specification has been debugged, it is translated into IF. Next, the back-ends of AVISPA run with IF file to identify the attacks. Figure 5 shows the simulation results of AVISPA for the RR-IBE and the proposed protocol. These results show that no revealed attacks are found and security goals are attained for the proposed protocol.

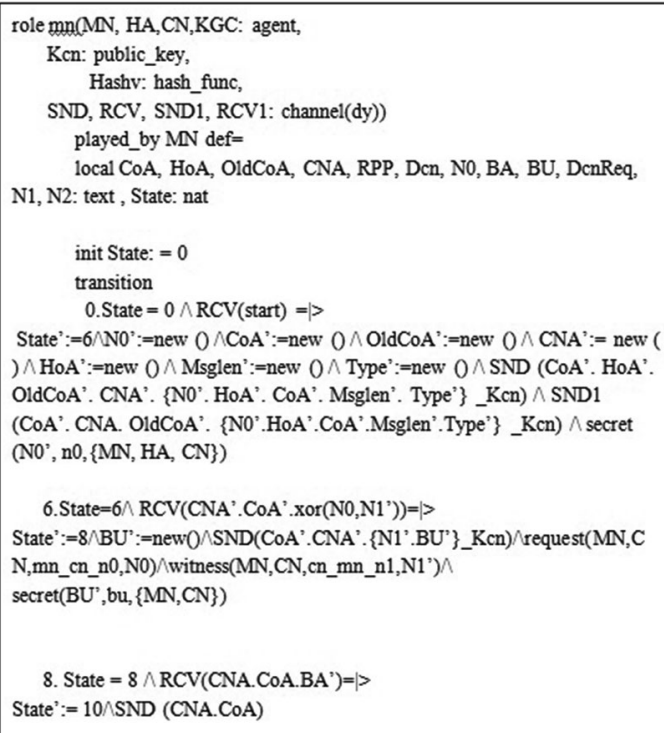

Figure 4. Role of a mobile node in AVISPA.

\section{Performance evaluation}

In this section, performance analysis of the proposed scheme with the other related works are presented in terms of communication payload and latency of the binding update.

\subsection{Message size}

The total size (in bytes) required for all messages is obtained during the run of the protocol. Here, the total message size computed for RRP, RR-IBE and the proposed protocol are 396, 1572 and 992 bytes, respectively. The size of messages can be calculated using the individual components of the protocol such as HOTI, COTI, BU and BA. For example, the message size of HOTI of the proposed protocol is computed as follows:

Message size of HOTI $=5 * 128+1024=1664$ bits. Total no of bytes required for each step of the proposed protocol $=$ HOTI + HOTI' + COTI + PPKReq + PPKRes $+\mathrm{AU}+\mathrm{BU}+\mathrm{BA}=1664+1408+408+320+512+$ $320+1280+256=7936$ bits $=992$ bytes .

By observing the total computations as shown in table 3 , the communication payload of the proposed protocol is less than RR-IBE and is higher than RRP because the security provided in RRP is less when compared to the proposed protocol.

\subsection{Latency of binding update}

Latency is the measure of the time consumed for a message to move from source to destination. The BU latency of the proposed protocol can be calculated using the system parameters (shown in table 4) obtained from the existing works [16-18]. The transmission time of any message can be computed as follows:

Transmission time $=$ Size of data/Bit rate.

The BU latency for the proposed protocol can be calculated as follows:

Total BU latency $=$ Time required at source for sending messages + Time required at intermediate nodes + Time required at destination for processing the messages. The computation of the latency is as follows:

$$
\begin{aligned}
& \text { Latency between } \mathrm{MN} \text { and } \mathrm{HA}=3.332+2.768 \\
& \quad+0.5=6.664 \mathrm{~ms} \\
& \text { Latency between } \mathrm{MN} \text { and } \mathrm{CN}=7.34+9.628+6.288 \\
& \quad=23.256 \mathrm{~ms}
\end{aligned}
$$

\subsection{Communication payload}

The communication payload includes the length of the header and the message content. Figures 6 and 7 show the 


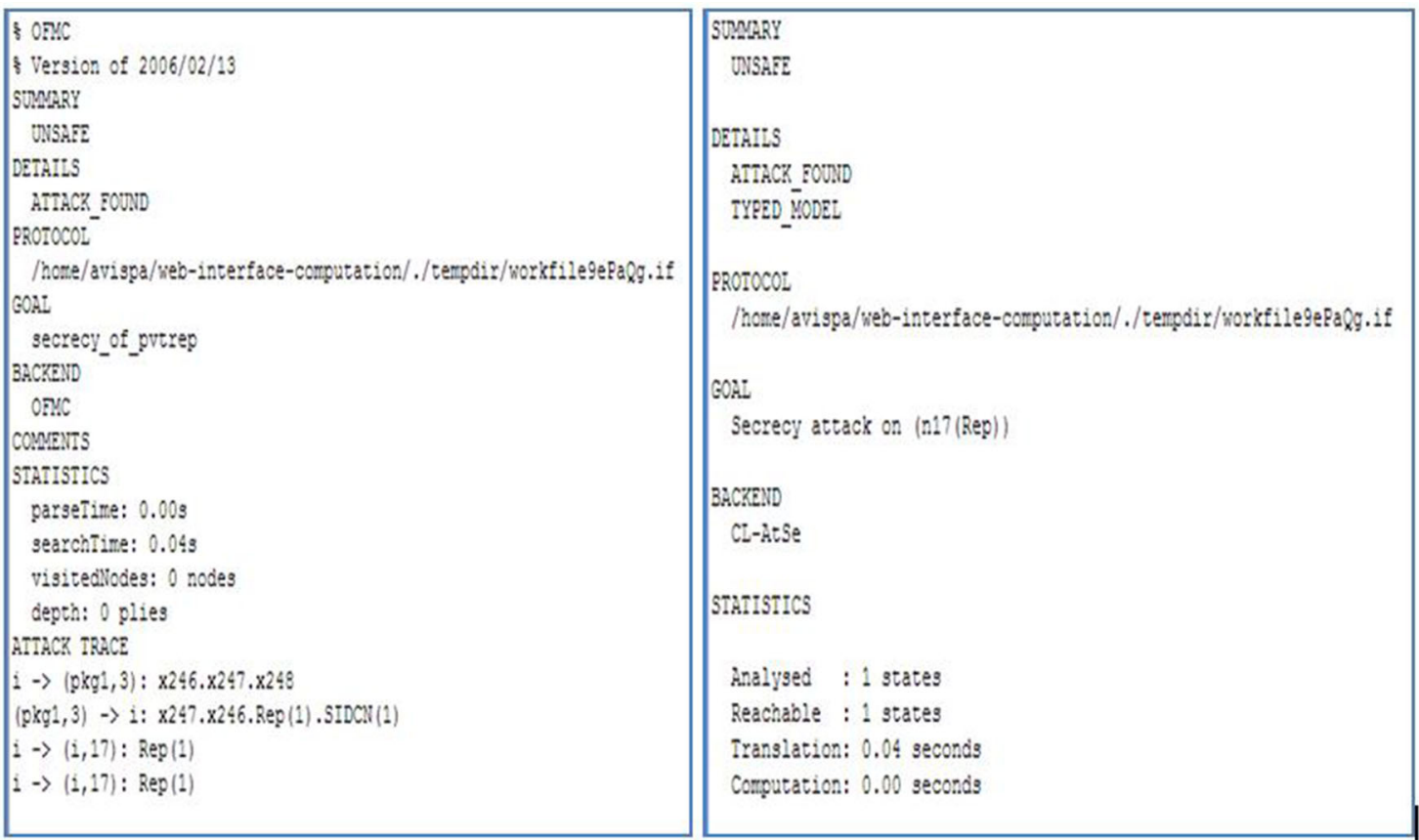

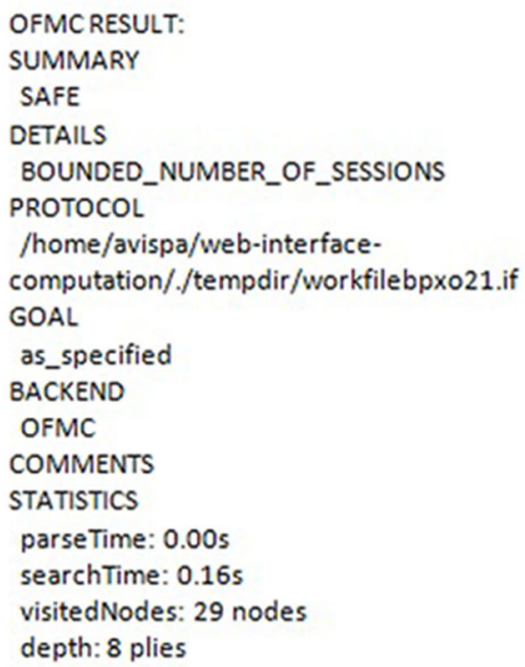

(a)

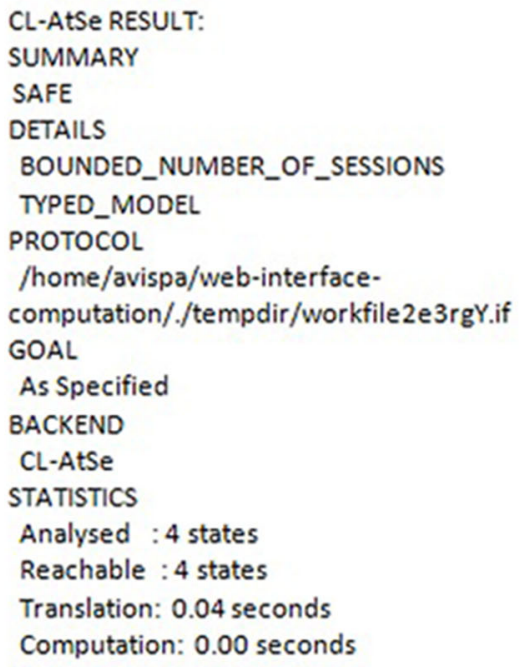

(b)

Figure 5. (a) OFMC and CL-AtSe results of RR-IBE. (b) OFMC and CL-AtSe results of proposed protocol.

comparison between the proposed protocol and the existing protocols in terms of communication payload between $\mathrm{MN}$ and $\mathrm{CN}$ through $\mathrm{HA}$ and directly between $\mathrm{MN}$ and $\mathrm{CN}$.

From the observations of both cases, it is seen that the proposed protocol provides less cost for communication payload of the BU messages when compared to RR-IBE. It is because of the following reasons in the proposed protocol: (i) the requirement for a pair of keys to get the CN's private key is eliminated as the $\mathrm{CN}$ receives only the partial private key from the $\mathrm{KGC}$ and not the complete private key; (ii) The contents of the messages are reduced; and (iii) light-weight encryption is done using CLPKE, which does not require any pairing operations. However, the proposed protocol incurs higher cost than RRP in case of communication payload between $\mathrm{MN}$ and $\mathrm{CN}$ but it provides better security. 
Table 3. Size of messages.

\begin{tabular}{lcccr}
\hline & \multicolumn{4}{c}{ Message size for single BU (in bytes) } \\
\cline { 2 - 5 } Protocol & MN-HA-CN & MN-CN & CN-key generator & Total \\
\hline RRP & 192 & 204 & - & 396 \\
RR-IBE & 672 & 700 & 200 & 1572 \\
Proposed & 384 & 408 & 104 & 992 \\
\hline
\end{tabular}

Table 4. System parameters.

\begin{tabular}{lc}
\hline Processing time in HA,CN and MN & $0.5 \mathrm{~ms}$ \\
Propagation time in wireless links & $2 \mathrm{~ms}$ \\
Bit rate in wireless links & $2 \mathrm{Mbps}$ \\
Propagation time in wired links & $0.5 \mathrm{~ms}$ \\
Bit rate in wired links & $100 \mathrm{Mbps}$ \\
SHA operation on MN & $0.019111 \mathrm{~ms}$
\end{tabular}

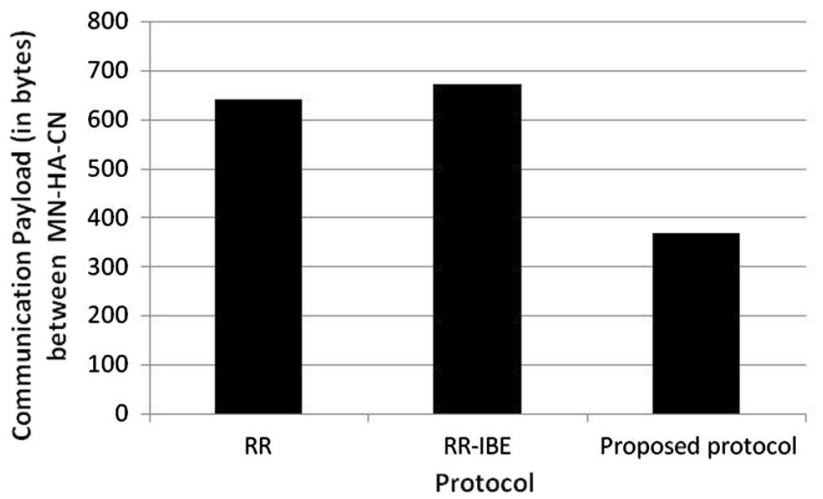

Figure 6. Communication payload between $\mathrm{MN}$ and $\mathrm{CN}$ through HA (in bytes).

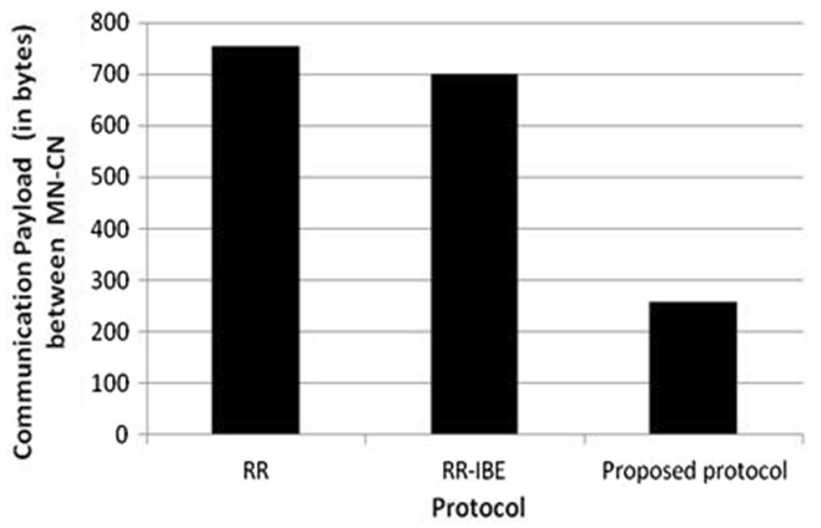

Figure 7. Communication payload between $\mathrm{MN}$ and $\mathrm{CN}$ (in bytes).

Figure 8 shows comparison results of the proposed protocol with the existing protocols in terms of the processing time (in $\mathrm{ms}$ ).

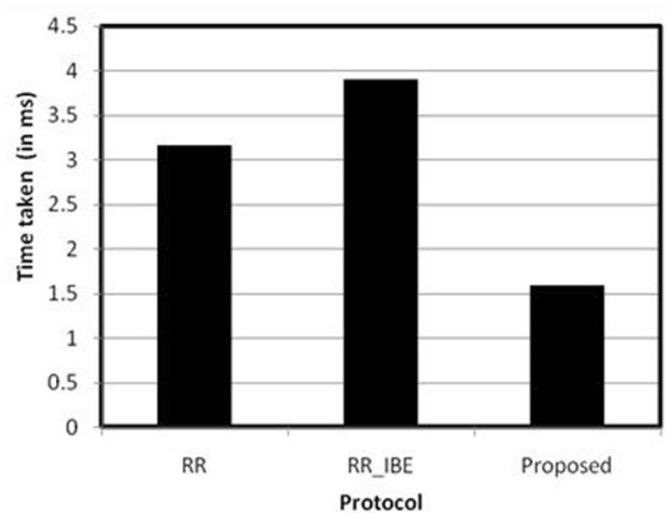

(a)

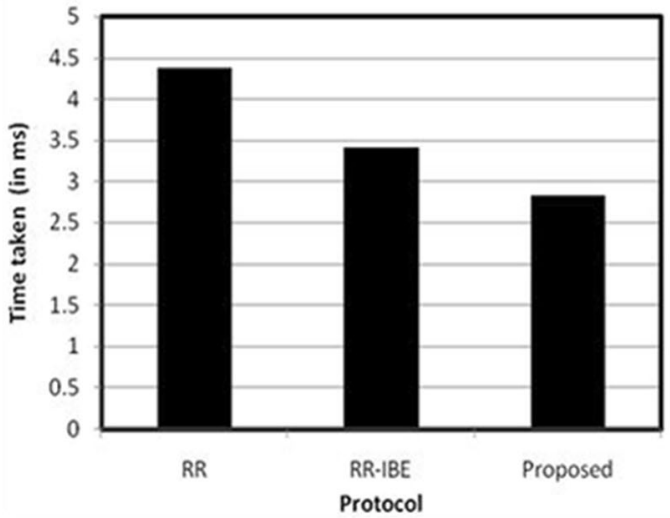

(b)

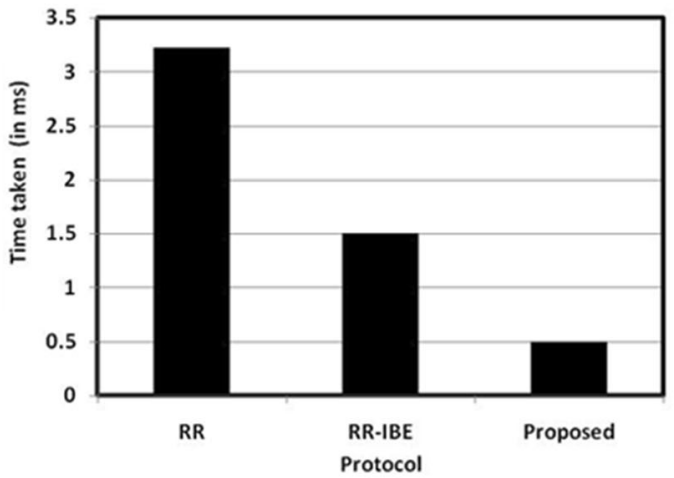

(c)

Figure 8. (a) Processing time at source MN. (b) Processing time at intermediate between $\mathrm{MN}$ and HA. (c) Processing time at destination HA.

Figure 8a shows that the time taken by the proposed protocol at source MN is less than that of RR-IBE. Here, the processing time at source depends on the transmission time of the messages P1 and M1 for RR-IBE and RR, respectively. Similarly, the time taken (shown in figure $8 \mathrm{~b}$ ) at intermediate for the proposed protocol is less than RRIBE. Figure $8 \mathrm{c}$ illustrates that the time taken at destination HA for the proposed protocol. Subsequently, both transmission time and computational time are also taken into account for computing the total latency. 


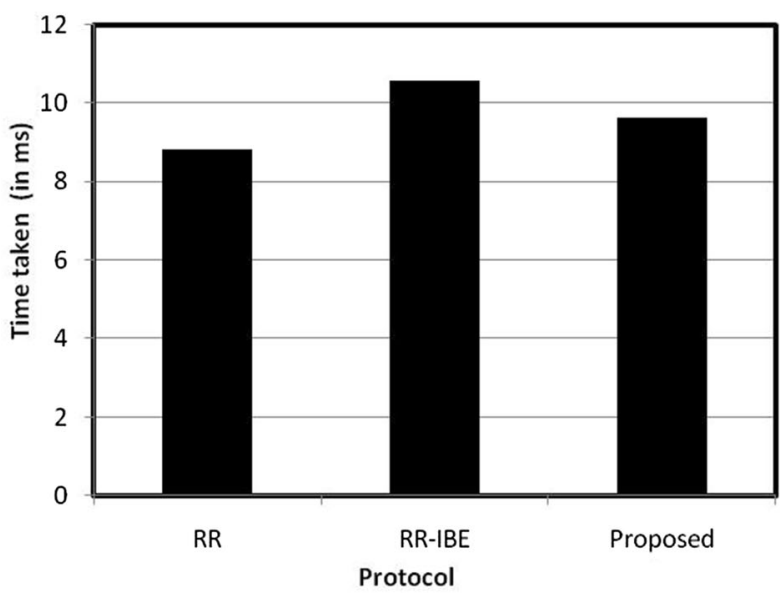

(a)

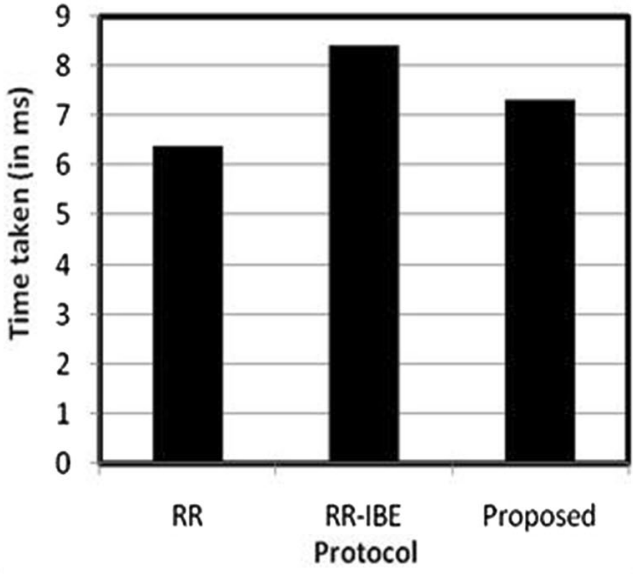

(b)

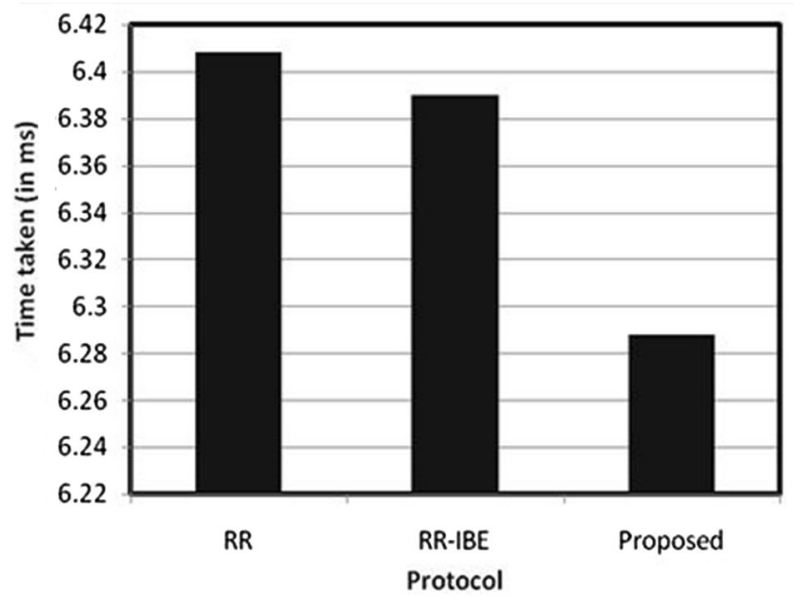

(c)

Figure 9. Computational latency (in $\mathrm{ms}$ ) between $\mathrm{MN}$ and $\mathrm{CN}$ : (a) at $\mathrm{MN},(\mathbf{b})$ between $\mathrm{MN}$ and $\mathrm{CN}$ and (c) at $\mathrm{CN}$.

Figure 9 demonstrates the comparison analysis of total latency at $\mathrm{MN}$ between the intermediate pair $\mathrm{MN}-\mathrm{CN}$ and at HA in the proposed and existing works. From figure 9a and $\mathrm{b}$, it is observed that the latency at intermediate and source for the proposed protocol is more than RRP but the latency at destination by the proposed protocol is less than RRP. But it provides enhanced security when compared to RRP. Evidently, the performance of the proposed protocol stands out among the other protocols because the proposed protocol uses few messages from $\mathrm{MN}$ to $\mathrm{HA}$ or to $\mathrm{CN}$ along with significant reduction in computation time for cryptographic operations.

\section{Conclusion}

In this paper, an enhanced Return Routability Protocol is proposed using CLPKE in mobility of IPv6. It provides authentication, confidentiality, integrity and possible attack preventions. In addition, the formal validation is verified using AVISPA for attack trace. No revealed attacks such as MITM, replay, amplification and false BU are found from the results of back-ends of AVISPA when compared to RRIBE. The performance evaluation of the proposed protocol illustrates that the new approach reduces the latency when compared to the existing RRPs.

\section{References}

[1] Al-Surmi I, Othman M and Ali B M 2012 Mobility management for IP-based next generation mobile networks: review, challenge and perspective. J. Netw. Comput. Appl. 35: $295-315$

[2] Mathi S K and Valarmathi M L 2015 A secure and efficient binding update scheme with decentralized design for next generation IP mobility. In: Artificial Intelligence and Evolutionary Algorithms in Engineering Systems, Springer, India, pp. 423-431 
[3] Modares H, Moravejosharieh A, Lloret J and Salleh R 2014 A survey of secure protocols in mobile IPv6. J. Netw. Comput. Appl. 39: 351-368

[4] Mathi S K and Valarmathi M L 2013 An efficacious and secure registration for internet protocol mobility. Defence Sci. J. 63(5): 502-507

[5] Arkko J, Vogt C and Haddad W 2007 Enhanced Route Optimization for Mobile IPv6, RFC4866

[6] Han Y H and Hwang S H 2006 Care-of address provisioning for efficient IPv6 mobility support. Comput. Commun. 29(9): $1422-1432$

[7] Ren K, Lou W, Zeng K, Bao F, Zhou J and Deng R H 2006 Routing optimization security in mobile IPv6. Comput. Netw. Elsevier 50(13): 2401-2419

[8] Vogt C, Bless R, Doll M and Kuefner T 2005 Early binding updates for mobile IPv6. In: Proceedings of International Conference on Wireless Communications and Networking. 3, pp. 1440-1445

[9] Yoon H S, Kim R H, Hong S B and Youm H Y 2006 PAKbased binding update method for mobile IPv6 route optimization. In: International Conference on Hybrid Information Technology, 2, pp. 617-623

[10] Alsalihy W A A and Alsayfi M S S 2013 Integrating identitybased encryption in the return routability protocol to enhance signal security in mobile IPv6. Wireless Personal Commun. 68(3): 655-669

[11] Barreto P S, Kim Y, Lynn B and Scott M 2002 Efficient algorithms for pairing-based cryptosystems. In: Proceedings of International Conference on Advances in Cryptology, Springer, Berlin Heidelberg, pp. 354-369

[12] Wang F and Zhang Y 2008 A new provably secure authentication and key agreement mechanism for SIP using certificateless public-key cryptography. Comput. Commun. 31(10): 2142-2149

[13] Zhang Z, Wong D S, Xu J and Feng D 2006 Certificateless public-key signature: security model and efficient construction. In: Proceedings of International Conference on Applied Cryptography and Network Security. Springer, Berlin Heidelberg, pp. 293-308

[14] AVISPA Team, 2013, AVISPA v1.1 user manual

[15] Pura M, Patriciu V and Bica I 2009 Modeling and formal verification of implicit on-demand secure ad hoc routing protocols in HLPSL and AVISPA. Int. J. Comput. Commun. 2(3): 25-32

[16] Argyroudis P G, Verma R, Tewari H and O'Mahony D 2004 Performance analysis of cryptographic protocols on handheld devices. In: Proceedings of 3rd IEEE International Symposium on Network Computing and Applications. pp. 169-174

[17] Hess A and Shafer G 2002 Performance evaluation of AAA/ Mobile IP Authentication. In: Proceedings of 2nd International Symposium on Polish-German Teletraffic, Gdansk, Poland

[18] McNair J, Akyildiz I F and Bender M D 2000 An intersystem handoff technique for the IMT-2000 system. In: Proceedings of International Computer and Communications. 1: 208-216 